\title{
Homogeneous selection promotes microdiversity in the glacier-fed stream
}

\section{microbiome}

3 Stilianos Fodelianakis ${ }^{1 *}$, Alex D. Washburne ${ }^{2,3}$, Massimo Bourquin ${ }^{1}$, Paraskevi Pramateftaki ${ }^{1}$,

4 Tyler J. Kohler ${ }^{1}$, Michail Styllas ${ }^{1}$, Matteo Tolosano ${ }^{1}$, Vincent De Staercke ${ }^{1}$, Martina Schön ${ }^{1}$,

5 Susheel Bhanu Busi ${ }^{4}$, Jade Brandani ${ }^{1}$, Paul Wilmes ${ }^{4}$, Hannes Peter ${ }^{1} \&$ Tom J. Battin ${ }^{1 *}$

$7 \quad{ }^{1}$ Stream Biofilm \& Ecosystem Research Lab, ENAC Division, Ecole Polytechnique Federale de

8 Lausanne

$9 \quad{ }^{2}$ Department of Microbiology and Immunology, Montana State University, USA

$10{ }^{3}$ Selva Analytics, LLC, Bozeman, Montana, USA

$11{ }^{4}$ Systems Ecology Research Group, Luxembourg Centre for Systems Biomedicine, University of

12 Luxembourg, Esch-sur-Alzette, Luxembourg

13

14 *Address correspondence to: Tom J. Battin; tom.battin@epfl.ch, Stilianos Fodelianakis;

15 stylianos.fodelianakis@epfl.ch

16

17 Running title: homogeneous selection promotes microdiversity 
19 Microdiversity, the organization of microorganisms into groups with closely related but

20 ecologically different sub-types, is widespread and represents an important linchpin between

21 microbial ecology and evolution. However, the drivers of microdiversification remain largely

22 unknown. Here we show that selection promotes microdiversity in the microbiome associated

23 with sediments in glacier-fed streams (GFS). Applying a novel phylogenetic framework, we

24 identify several clades that are under homogeneous selection and that contain genera with higher

25 levels of microdiversity than the rest of the genera. Overall these clades constituted $\sim 44 \%$ and

$26 \sim 64 \%$ of community $\alpha$-diversity and abundance, and both percentages increased further in GFS

27 that were largely devoid of primary producers. Our findings show that strong homogeneous

28 selection drives the microdiversification of specialized microbial groups putatively underlying

29 their success in the extreme environment of GFS. This microdiversity could be threatened as

30 glaciers shrink, with unknown consequences for microbial diversity and functionality in these

31 ecosystems. 


\section{Introduction}

Microdiversity, the organization of microbial sub-taxa with distinct niches within a larger

34 phylogenetic clade ${ }^{1}$, is an intrinsic property of microbial communities that is widely distributed

35 in nature. Whereas initial studies suggested that microdiversity results from genetic drift and

36 does not necessarily involve phenotypic differences ${ }^{2,3}$, such differences are now well

37 documented in major biomes of the planet. In marine environments, microdiversity within

38 marine picocyanobacteria (e.g., Prochlorococcus, Synechococcus) and heterotrophic bacteria

39 (e.g., Vibrio, Pelagibacter) includes sub-taxa acclimated to light intensity, nutrient availability

40 and hydrostatic pressure ${ }^{4-9}$. In freshwaters, members of the ubiquitous Limnohabitans exhibit

41 microdiversity related to temperature and nutrient availability ${ }^{10}$, while microdiversity has also

42 been reported from Streptomyces and Curtobacterium in soils along climatic ${ }^{11}$ and latitudinal $^{12}$

43 gradients, respectively.

44 Microdiversity results in phenotypic (i.e., trait) differentiation, and traits affect the

45 fitness and performance of a taxon under specific environmental conditions ${ }^{1,13}$. Therefore, from a

46 community ecology perspective, it is intuitive to assume that microdiversity relates to the

47 assembly process of selection, i.e., deterministic fitness differences among species ${ }^{14}$. Recent

48 microbial community surveys tend to support this notion, for instance by observing different

49 temporal turnover patterns among marine sub-taxa ${ }^{15,16}$ or by examining the distribution of

50 microbial sub-taxa along environmental gradients ${ }^{17}$ and their participation in different biotic

51 interactions ${ }^{18}$. However, it is yet unclear whether there is a causal relationship between the

52 community assembly process of selection (henceforth referred to as "selection") and

53 microdiversity (i.e., whether selection promotes microdiversity). Here we posit that such a

54 relationship exists between microdiversity and selection, the latter being expanded to include 
55 fitness differences within species as well, given that bacterial species boundaries are blurry ${ }^{19}$.

56 More specifically, we hypothesize that this relationship should be more prevalent in ecosystems

57 where community assembly is largely driven by selection. Under the assumption of phylogenetic

58 conservatism (i.e., closely related taxa share similar phenotypes), taxa with high fitness in such

59 ecosystems should form coherent phylogenetic clades. Within these clades, closely related taxa

60 with a specific fitness advantage may occupy diverse niches via fine-tuning or gain of novel

61 phenotypic traits ${ }^{1}$. Eventually, this would foster the generation of microdiversity within clades

62 under selection. Hence, empirical evidence showing a relationship between clades under

63 selection and microdiversity in an ecosystem dominated by selection would suggest that

64 selection fosters microdiversification.

65 Phylogenetic clades under selection could be identified by expanding the analytical

66 framework that is used to infer community-wise assembly processes ${ }^{20,21}$. At the community

67 level, lower phylogenetic turnover than expected by chance indicates communities under

68 homogenous selection ${ }^{20,21}$. Homogeneous selection indicates that the same selective processes

69 (e.g., abiotic conditions or biotic interactions) are acting among the studied communities, and it

70 appears to dominate community assembly in extreme, energy-limited ecosystems ${ }^{22-24}$. In

71 analogy, the presence of phylogenetically similar sequence variants (SVs) occurring more

72 frequently than expected by chance could be used to identify phylogenetic clades under

73 homogeneous selection.

74 Glacier-fed streams (GFS) seem well suited to test the potential relationship between

75 microdiversity and selection, because of their extreme environment (e.g., low temperature,

76 oligotrophic conditions, hydraulic stress) and diverse communities of microbes and

77 macroorganisms ${ }^{25-27}$. We expect that because of the extremeness in the GFS environment, 
78 homogenous selection results in the prevalence of a few highly specialized phylogenetic clades

79 that are characterized by enhanced microdiversity. To that end we studied sediment biofilms at

80 twenty GFS across a 340-km long transect in the Southern Alps in New Zealand (Fig. S1). Our

81 sampling design allowed us to capture patterns of community turnover over a large spatial scale

82 as well as within streams (two reaches per stream and three biological triplicates per reach)

83 where dispersal should be more important and could potentially attenuate selection with mass

84 effects $^{21,28}$ via the water flow. Subsequently, we quantified the community assembly processes,

85 developed a novel framework to identify phylogenetic clades under selection and examined if

86 they are characterized by a high degree of microdiversity. 


\section{Results}

\section{Homogeneous selection is the dominant assembly process at the community level.}

89 Using a community-level framework ${ }^{20,21}$, we first examined the processes that govern the

90 assembly of sediment biofilm communities among and within the GFS (Methods). We found that

91 homogeneous selection (reflected as $\beta$ NTI values $<-2$ ) was the dominant assembly process for

$9289.2 \%$ of the community pairs among GFS (Fig. 1). Moreover, homogeneous selection

93 dominated (in $99.3 \%$ of the community pairs) the assembly within GFS, indicating that it was

94 not attenuated by downstream dispersal via water flow. Dispersal limitation drove assembly for

$959.5 \%$ of community pairs among GFS and its probability of occurrence increased with increasing

96 geographic distance between the compared communities (logistic regression, $\mathrm{z}=11.97, \mathrm{p}<$

97 0.001). Finally, variable selection and homogenizing dispersal drove assembly for $0.6 \%$ and

$980.25 \%$ of community pairs among GFS, respectively, while no single dominant process was

99 found in $0.45 \%$ of community pairs. 


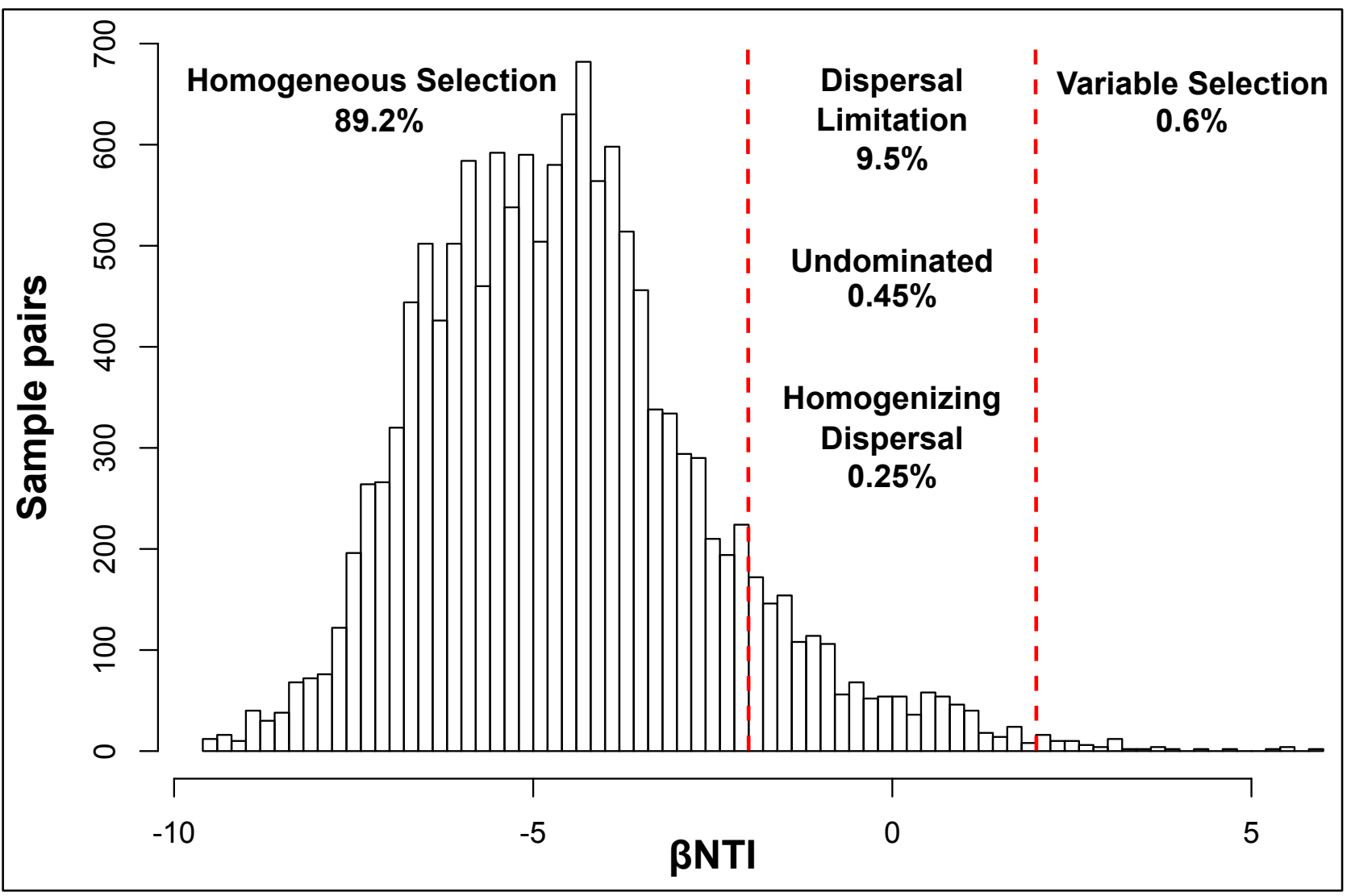

101 Figure 1. Homogeneous selection is the dominant assembly process at the community level. The

102 histogram shows the distribution of $\beta$ NTI values for sample comparisons across GFS and the proportion

103 of sample pairs under each community assembly processes. Vertical dashed red lines are drawn at $\beta$ NTI

104 values of -2 and +2 , which are the cutoff values for lower and higher than expected phylogenetic

105 community turnover, respectively, the former indicating homogeneous selection and the latter indicating

106 variable selection. The assembly processes governing the sample pairs in between are estimated from

107 compositional turnover patterns based on the $\mathrm{RC}_{\text {Bray }}$ index.

Phylogenetic clades under homogeneous selection are diverse, abundant and widespread.

Next, having confirmed the dominant role of selection in driving assembly at the

111 community level, we developed and applied a method that leverages null phylogenetic modeling

112 to identify phylogenetic clades that are under homogeneous selection. In analogy to the

113 community-level framework, we defined these clades as phylogenetically coherent groups that 
114 contain SVs with phylogenetically closer relatives across communities than expected by chance.

115 To identify such SVs, we used a z-score that describes how phylogenetically distant an SV in

116 one community is to its closest relative in another community compared to what is expected by

117 chance. Specifically, the z-score counts this phylogenetic distance in standard deviations with

118 respect to a null distribution of phylogenetic distances so that negative z-scores represent shorter

119 phylogenetic distances than expected by chance and vice versa. We then calculated the total z-

120 scores for each SV across the dataset, i.e., the sum of the z-scores of that SV across all

121 community pairs, excluding sample pairs from replicates of the same reach. For a given SV,

122 highly negative total z-scores indicate that it is replaced by closely related SVs in many

123 community pairs. In the presence of a phylogenetic signal at short phylogenetic distances, which

124 we verified for our dataset (Methods, Fig. S2), this indicates that the given SV has increased

125 fitness in the specific ecosystem, because it and its functionally similar close relatives are

126 widespread. Consequently, we used phylofactorization ${ }^{29,30}$ to identify phylogenetically coherent

127 groups of SVs that have significantly different total z-scores compared to outgroups.

128 We identified eight phylogenetic clades with significantly lower total z-scores compared

129 to outgroups (contrast tests, 3.3E-16 $<\mathrm{p}<6.8 \mathrm{E}-255$ ), comprising of 5 to $1418 \mathrm{SVs}$ each (Fig. 2,

130 Table S1). The consensus taxonomy of the largest identified clade (1418 SVs) affiliated to

131 Betaproteobacteria (Fig. 2). This clade also contained three sub-clades with distinctly low scores

132 and with consensus taxonomies affiliated to the family Comamonadaceae (575 SVs), to the

133 uncultured order Ellin6067 (54 SVs) and to the genus Methylotenera (48 SVs). The second

134 largest clade (602 SVs) had a consensus taxonomy affiliated to Alphaproteobacteria and it

135 contained a low-score sub-clade (5 SVs) affiliated to the genus Novosphingobium. The third

136 largest clade (338 SVs) was affiliated to the candidate class Saprospirae within Bacteroidetes 
137 while the smallest clade (18 SVs) was taxonomically affiliated to the genus Nitrospira.

138 Importantly, we did not identify any phylogenetic clade with significantly higher total z-scores

139 than expected by chance; this reflects the low contribution of heterogeneous selection in

140 governing assembly at the community level (i.e., low percentage of community pairs with higher

141 than expected phylogenetic turnover; Fig. 1).

142

143 


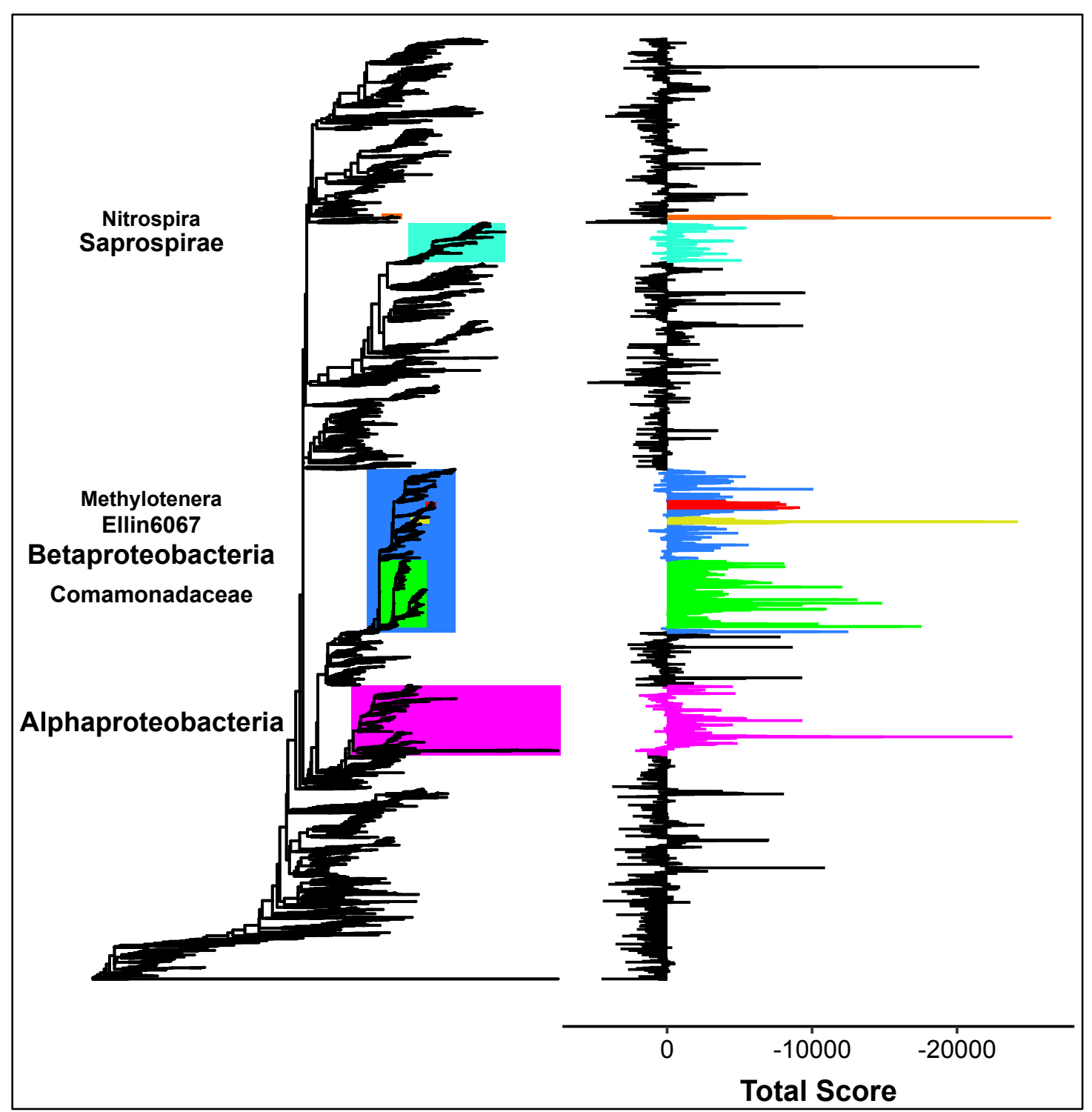

147 compared to outgroups. Identified clades with more than 15 SVs are color-coded on the phylogenetic

148 tree, and the consensus taxonomy is given for each clade on the left with font size proportional to

149 taxonomic depth. Clades nested within Betaproteobacteria are colored individually. The total score of

150 each SV (i.e., the sums of the z-scores across community pairs) is shown to the right as bars with colors

151 matching the clades' colors. 
We found that these clades contained a significant part of the total bacterial diversity and

154 abundance at all GFS, with on average $43.7 \%(25.5 \%$ to $61.6 \%)$ of the total SVs and $64 \%(37.6$

155 to $83.3 \%$ ) of the total sequences per sample. Additionally, there was a notable overlap between

156 the identified clades and the core microbiome defined as the bacterial genera present at all

157 reaches (Table S2, Supplementary Results). More specifically, nine of the twelve core genera

158 resided within the identified phylogenetic clades (Fig. 3); these genera included the majority of

159 the SVs $(59.5 \%)$ and of the sequences $(87.7 \%)$ present in the core genera. Furthermore, both the

160 abundance and the $\alpha$-diversity of the identified clades increased disproportionately compared to

161 the rest of the microbiome as sediment chlorophyll $a$ decreased (linear models, $\mathrm{n}=119$, adjusted

$162 \mathrm{R}^{2}=0.298$ and 0.302 , respectively, and $\mathrm{p}<0.001$ for both models) (Fig. 4A-B). Since sediments

163 with lower chlorophyll $a$ also contained fewer total bacterial cells (Pearson correlation, $\mathrm{r}=0.85$,

$164 \mathrm{p}<0.001$ ) (Fig. 4C), the above correlations held true with decreasing cell numbers as well (linear

165 models, $\mathrm{n}=119$, adjusted $\mathrm{R}^{2}=0.282$ and 0.252 , respectively, and $\mathrm{p}<0.001$ for both models) (Fig.

166 S3). Collectively, these results indicate that the identified clades are ecologically successful in

167 the extreme GFS environment, underscoring their apparent fitness and corroborating that they

168 are under homogeneous selection. 


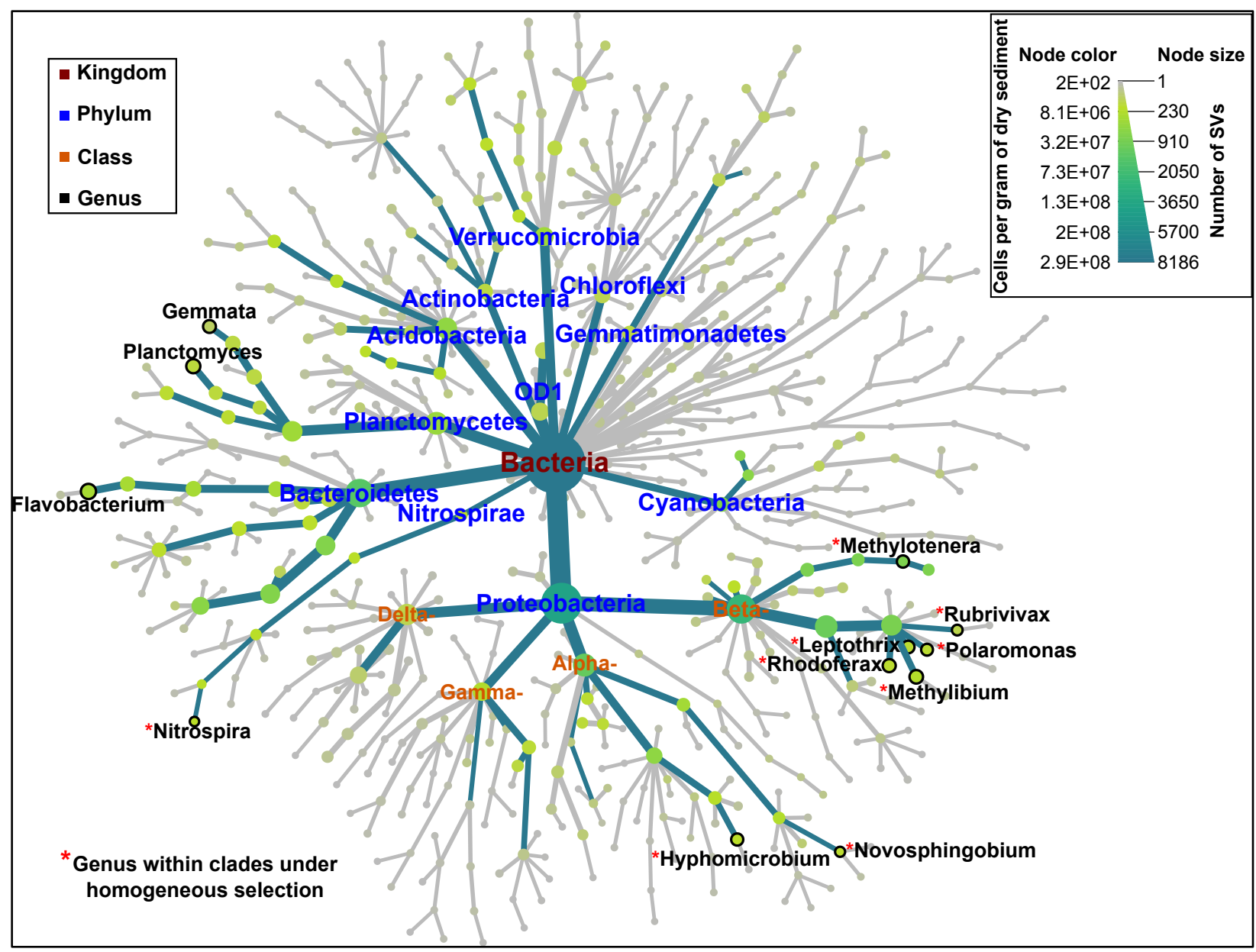

171 Figure 3. The core microbiome at the genus level and the phylogenetic clades under homogeneous

172 selection overlap highly. The overall core microbiome, i.e., taxonomic units found across all the sampled

173 GFS reaches, is represented as a hierarchy tree (dark green edges) within the overall taxonomic tree (dark

174 green and grey edges). The node color and size are proportional to the node's abundance (cells per gram

175 of dry sediment) and diversity (number of SVs), respectively, as per the legend on the upper right. Only

176 core genera, phyla, and classes within the Proteobacteria phylum are labeled to improve visualization with

177 colors according to the legend on the upper left. Red asterisks indicate genera that reside in phylogenetic

178 clades under homogeneous selection. 

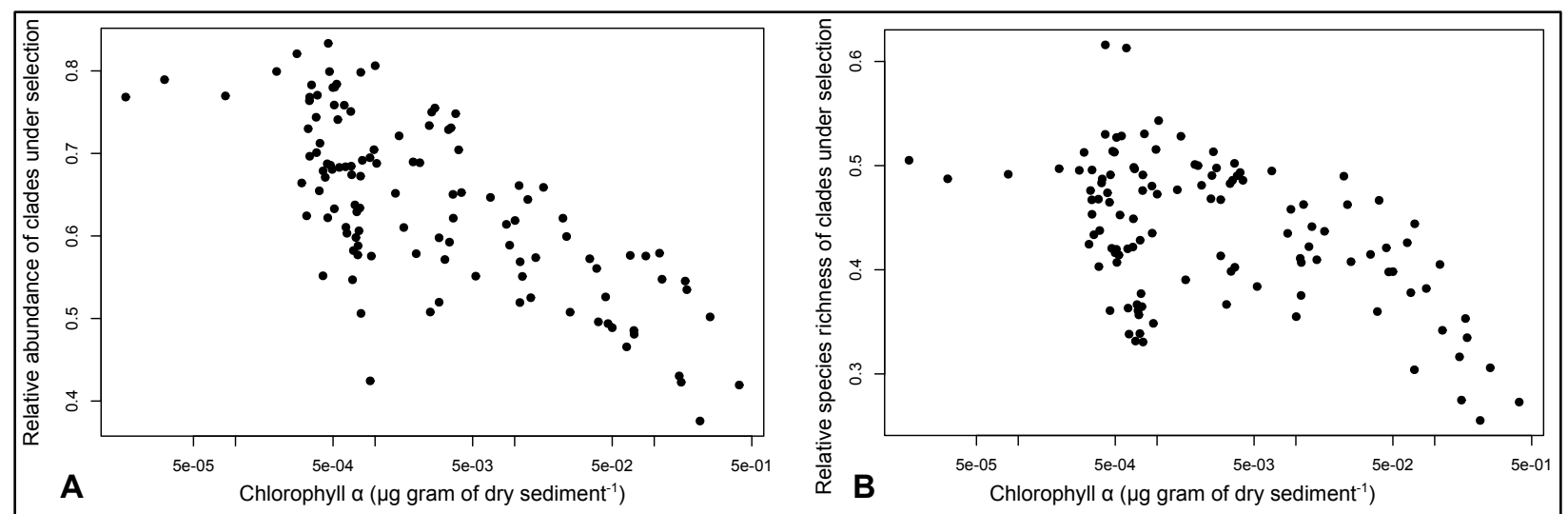

181 Figure 4. The phylogenetic clades under homogeneous selection thrive in sediments with low richness of the clades under homogeneous selection as a function of the sediment chlorophyll $a$. C. The total bacterial cell abundance as a function of the sediment chlorophyll $a$. For all panels, $\mathrm{n}=119$.

Eleven genera within phylogenetic clades under homogeneous selection are hotspots of 
192 compared similar taxonomic units, assessing the levels of microdiversity in genera within and

193 outside these clades. We restricted our analyses to genera with at least 2 SVs that are formally

194 assigned in the scientific nomenclature, resulting in the inclusion of 110 genera containing 2003

195 SVs in total. This represented approximately half (47.2\% on average, $26.5-79 \%$ per sample) of

196 the total sequences. Phylogenetic clades under homogeneous selection included 41 genera,

197 whereas 69 genera resided outside these clades (Table S3). Within-genera, microdiversity should

198 emerge as high numbers of closely related SVs. Moreover, if closely related SVs within genera

199 indeed occupy distinct niches, this should result in a wide genus spatial distribution. We

200 compared the following three attributes of all genera: the number of SVs per genus, the mean

201 nucleotidic similarity and the spatial distribution (i.e., the B parameter sensu Levins ${ }^{31}$ ) as a proxy

202 for niche breadth given that there was no single environmental gradient driving $\beta$-diversity

203 (Methods) (Supplementary Results, Fig. S4).

204 Our results-revealed eleven genera, namely Methylotenera, Rhodoferax, Leptothrix,

205 Polaromonas, Methylibium, Rubrivivax, Thiobacillus, Novosphingobium, Hyphomicrobium,

206 Rhodobacter and Nitrospira, with disproportionately higher levels of microdiversity compared to

207 other genera (Fig. 5). Strikingly, all these genera resided within clades under homogeneous

208 selection and represented a large part of the SVs (33\%) and of the cells (average: 55.5\%; range:

$20935.1-78 \%$ ) therein. Furthermore, these genera had high numbers of SVs per genus (average:

210 71.9; range: 20-130), as well as high mean pairwise nucleotidic similarity (average: 96.1\%;

211 range: 95.1-96.7\%) and large mean niche breadths (average: 5.96; range: 4.62-8.12) (Fig. 5).

212 Specifically, they had approximately 10 to 50-fold higher numbers of SVs per genus compared

213 to other genera, as well as higher mean pairwise nucleotidic similarity (t-tests, $\mathrm{p}<<0.001$ for all

214 comparisons) and niche breadths (t-tests, 6.1E-05 $<\mathrm{p}<0.04$ ) compared to the species-rich 
215 genera of Flavobacterium, Planctomyces, Gemmata and Bdellovibrio that do not reside within

216 phylogenetic clades under homogeneous selection (Fig. 5). Overall, our results show that the

217 eleven identified genera were the only genera across the dataset that simultaneously had high

218 numbers of SVs (per genus), high mean pairwise nucleotidic similarity and wide spatial

219 distribution. This is evidence that microdiversity is disproportionally more present within

220 phylogenetic clades under homogeneous selection. 


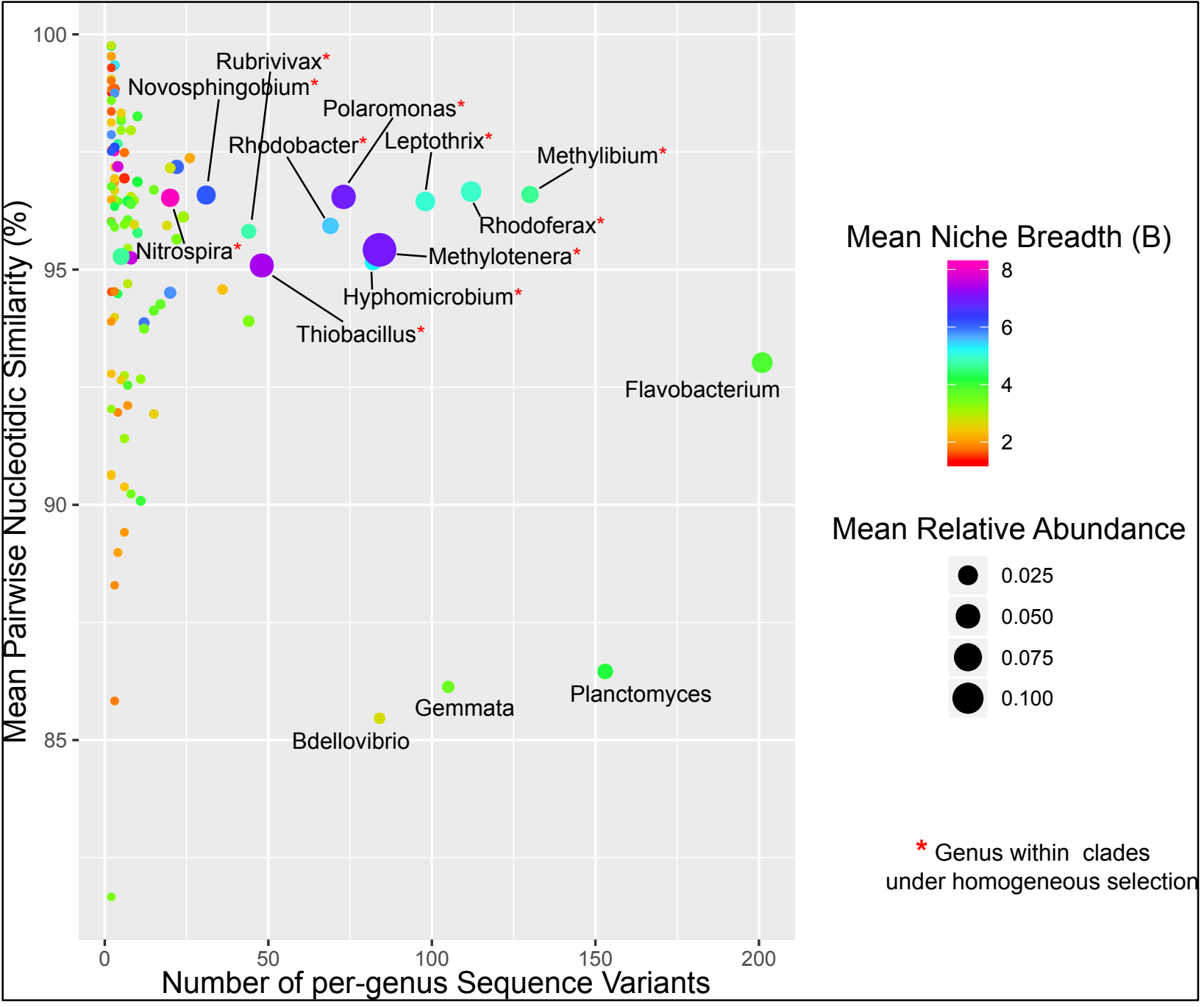

226 Figure 5. Microdiversity is disproportionately more present in genera residing within phylogenetic

227 clades under homogeneous selection. The plot shows the per-genus degree of microdiversification,

228 assessed via the number of per-genus Sequence Variants (SVs), the mean pairwise nucleotidic similarity

229 and the mean niche breadth ( $\mathrm{B}$ - color coding). Dot sizes are proportional to the mean relative abundance.

230 Labels are shown only for genera with more than $50 \mathrm{SVs}$, or for the eleven identified genera within

231 phylogenetic clades under selection, the latter labeled with an additional red asterisk. Note the high

232 number of per-genus SVs of Flavobacterium, Planctomyces, Gemmata and Bdellovibrio, which do not

233 reside within clades under homogeneous selection. 


\section{SVs with low phylogenetic turnover foster microdiversity within specific genera}

Finally, we asked whether the eleven genera with elevated microdiversity are also

237 characterized by disproportionately low phylogenetic turnover (indicative of strong

238 homogeneous selection). In other words, if selection promotes microdiversity, the eleven genera

239 with the highest levels of microdiversity should also show the strongest signs of homogeneous

240 selection. More specifically, the eleven genera with the highest levels of microdiversity should

241 contain clusters of SVs with low phylogenetic turnover that are driving the increase of

242 microdiversity within the genus.

243 To test that, we first detected SVs with constantly low phylogenetic turnover (LPT-SVs)

244 and their phylogenetically closer-than-expected relatives (CR), to then examine if they are

245 primarily present within the eleven genera of interest. Subsequently, we examined if these

246 clusters are also characterized by high sequence similarity and high niche differentiation. We

247 defined LPT-SVs as SVs that have lower z-scores than expected by chance in the majority of the

248 applicable community comparisons (SVs having a median z-score < -2). LPT-SVs represent SVs

249 with a CR across most GFS and do not necessarily have high occupancies. For example, an LPT-

250 SV of the genus Methylibium in our dataset is only present in four reaches across all GFS, but

251 has three CRs (with which it is $99.7 \%$ similar at the sequenced part of the 16S rRNA gene on

252 average) in 204 out of the 344 applicable community pairs that span 33 out of the 40 reaches

253 across all GFS. In other words, these four SVs (the LPT-SV and its CRs) are highly similar

254 genetically and have a broad spatial distribution as a cluster but not individually. Taking spatial

255 distribution as a proxy for niche breadth and having already excluded the presence of dispersal

256 limitation, this pattern points towards the coverage of distinct niches by these sub-taxa. 
257 Therefore, a strong presence of LPT-SVs in the eleven genera would indicate that these genera

258 broaden their spatial distribution in GFS by microdiversification.

259 Indeed, we found that the clusters of the LPT-SVs and their CRs were disproportionally

260 present in the eleven genera and they had high genetic similarity and wide spatial distributions.

261 We identified a total of 172 LPT-SVs and found that their majority (143 SVs) resided within

262 phylogenetic clades under homogeneous selection. Furthermore, these LPT-SVs had very low

263 phylogenetic turnover, accounting for $80.5 \%$ of the total z-score among LPT-SVs (Table S4).

264 Importantly, $104(60.5 \%)$ of the LPT-SVs were taxonomically assigned to the eleven genera of

265 interest with nine out of these genera containing at least one LPT-SV (Table 1). The LPT-SVs

266 and their CRs had a mean nucleotidic similarity of 97.2-99.8\%, which was higher than that of the

267 respective genus. This indicates that they represent clusters of sub-taxa. These clusters had broad

268 spatial distributions, occupying 32 to 40 of the 40 total reaches across all GFS. In most of the

269 cases, except in Methylibium and in Rhodobacter that contained only 3 and 1 LPT-SV,

270 respectively, the reach occupancy of LPT-SVs and CRs was higher than that of the rest of the

271 SVs within the same genus. This indicates that the former contributes in widening the spatial

272 distribution of the respective genus. Moreover, LPT-SVs and CRs were highly abundant within

273 the respective genus (except in Methylibium and Rhodobacter), comprising 47.2-99\% of the total

274 cells affiliated with this genus. These results confirm that clusters of SVs with low phylogenetic

275 turnover are driving the increase of microdiversity in the eleven genera of interest, and suggest

276 that homogeneous selection promotes microdiversity in the examined GFS sediments. 
Table 1. The presence and properties of low phylogenetic turnover Sequence Variants (LPT-SVs)

281 and their phylogenetically closer than expected relatives (CR) in the eleven genera that reside

282 within clades under homogeneous selection and that have higher levels of microdiversity compared

283 to other genera.

\begin{tabular}{ccccccccc}
\hline Genus & \multicolumn{2}{c}{ Number of SVs } & \multicolumn{2}{c}{$\begin{array}{c}\text { Mean Nucleotidic } \\
\text { Similarity (\%) }\end{array}$} & Cell Ratio & $\begin{array}{c}\text { Occupancy } \\
\text { (Reaches) }\end{array}$ \\
\hline & Total & LPT & $\begin{array}{c}\text { Total CR } \\
\text { + LPT } \\
\text { (mean CR } \\
\text { per LPT) }\end{array}$ & $\begin{array}{c}\text { Among } \\
\text { LPT-CR }\end{array}$ & $\begin{array}{c}\text { Genus } \\
\text { mean }\end{array}$ & $\begin{array}{c}\text { (LPT+CR)/Total } \\
\text { Genus }\end{array}$ & LPT+CR & Rest* \\
\hline Nitrospira & 20 & 16 & $17(3.3)$ & 99 & 96.5 & 0.99 & $40 / 40$ & $6 / 40$ \\
Methylotenera & 84 & 29 & $46(4.7)$ & 99.1 & 95.4 & 0.736 & $40 / 40$ & $40 / 40$ \\
Polaromonas & 73 & 20 & $35(6.8)$ & 98.8 & 96.5 & 0.918 & $40 / 40$ & $38 / 40$ \\
Leptothrix & 98 & 19 & $37(8.5)$ & 99.3 & 96.4 & 0.733 & $40 / 40$ & $38 / 40$ \\
Novosphingobium & 31 & 8 & $15(7.5)$ & 97.2 & 96.6 & 0.924 & $40 / 40$ & $30 / 40$ \\
Rhodoferax & 112 & 5 & $21(7.2)$ & 99.1 & 96.7 & 0.472 & $40 / 40$ & $40 / 40$ \\
Thiobacillus & 48 & 3 & $9(2)$ & 99.3 & 95.1 & 0.629 & $32 / 40$ & $28 / 40$ \\
Rhodobacter & 69 & 1 & $2(1)$ & 99.8 & 95.9 & 0.051 & $30 / 40$ & $39 / 40$ \\
Methylibium & 130 & 3 & $4(1.7)$ & 99.7 & 96.6 & 0.136 & $33 / 40$ & $40 / 40$ \\
Rubrivivax & 44 & 0 & 0 & $\mathrm{n} / \mathrm{a}$ & 95.8 & $\mathrm{n} / \mathrm{a}$ & $\mathrm{n} / \mathrm{a}$ & $40 / 40$ \\
Hyphomicrobium & 82 & 0 & 0 & $\mathrm{n} / \mathrm{a}$ & 95.2 & $\mathrm{n} / \mathrm{a}$ & $\mathrm{n} / \mathrm{a}$ & $40 / 40$ \\
\hline
\end{tabular}




\section{Discussion}

289 GFS. Low phylogenetic turnover, attributed to homogeneous selection in community

290 ecology $^{20,21}$, dominated the assembly of microbial communities as is typical for energy-limited

291 environments ${ }^{22-24}$. Our analytical framework further allowed us to dissect the contribution of

292 individual phylogenetic clades to this low phylogenetic turnover. In analogy, we call these clades

293 as being under strong homogeneous selection, and both their high occupancies and abundances

294 in GFS corroborate this notion. Focusing on the eleven genera within these clades with high

295 microdiversity, we found that microdiversity was fostered by specific fine-scale clusters of SVs

296 with low phylogenetic turnover. These findings shed new light on the drivers of the fine-scale

297 phylogenetic architecture and its consequences for the success of microbial life in the extreme

298 GFS environment.

299 Our results further suggest that the phylogenetic clades under homogeneous selection can

300 successfully occupy a niche in the GFS that is largely devoid of algal primary producers. This is

301 indicated by their stronger presence in sediments with little chlorophyll $a$. Concomitantly, lower

302 cell abundance in these sediments further evokes that the rest of the microbiome is energy-

303 limited in these sediments. We interpret these patterns as evidence for an ecological niche

304 governed by chemolithotrophic rather than heterotrophic energy pathways, as is typical in

305 extreme environments, including the cryosphere and deep biosphere ${ }^{32-35}$.

306 This notion is indeed supported by the physiologies broadly studied and established for

307 the genera residing within the clades under homogeneous selection. For instance, the globally- 
308 spread $^{36}$ psychrophilic genus Polaromonas is facultatively chemolithotrophic and metabolically 309 versatile $^{37}$, and was even reported to be microdiverse ${ }^{38}$. Furthermore, the obligate methylotrophs

310 Methylibium, Methylotenera and Hyphomicrobium have been found in deglaciated alpine soils ${ }^{39}$

311 and glaciers ${ }^{40}$, and can utilize a diverse array of $\mathrm{C}_{1}$ compounds ${ }^{41-43}$ that can occur as

312 intermediates in methane oxidation typical for the sub-glacial environment ${ }^{44,45}$. The anoxygenic

313 phototrophs and nitrogen fixing genera Rhodobacter, Rubrivivax and Rhodoferax include

314 psychrotolerant isolates ${ }^{46,47}$ and have been found in ice cores ${ }^{48}$, deglaciated soils ${ }^{49}$ and

315 glaciers ${ }^{40,50}$. Furthermore, members of the Nitrospira genus are ubiquitous nitrite oxidizers, and

316 species able to perform complete ammonium oxidation have recently been reported in a high-

317 altitudinal and cold-water river $^{51}$. The sulfur-oxidizing, facultative anaerobe and

318 chemolithotrophic Thiobacillus has a sequenced genome from a subglacial isolate revealing cold

319 adaptations $^{52}$ and is frequently found in cold-related environments ${ }^{53,54}$. The only "classical"

320 heterotroph among the identified genera is the iron oxidizing Leptothrix ${ }^{55}$, which has been

321 recently reported from a metagenome from Antarctica ${ }^{56}$.

322 The environment of GFS is predicted to change dramatically as glaciers shrink owing to

323 climate change ${ }^{25,57}$. A recent synthesis has suggested that specialist species that are well adapted

324 to the glacial conditions in GFS are highly threatened by glacier retreat ${ }^{25}$. At the same time, as

325 turbidity decreases in GFS because of reduced discharge and sediment loads, the environment

326 will become more advantageous for primary production ${ }^{57}$. Therefore, the ecological niche with

327 its microdiverse clades that we have identified will most likely vanish with ongoing glacier

328 shrinkage, and with this, a hidden biodiversity that has adapted to the GFS environment and that

329 could even contain unexploited potential for biotechnology ${ }^{58}$. 
Apart from highlighting the importance of microdiversity, our study also contributes in

331 understanding the mechanisms controlling community assembly under the umbrella of

332 deterministic and stochastic processes, which is a debated topic in microbial ecology ${ }^{59,60}$.

333 Analytical frameworks detecting and quantifying assembly processes at the community

334 level $^{20,21,61,62}$ have provided useful insights in a great variety of ecosystems ${ }^{60}$. Recently, the focus

335 has expanded to the identification of specific components of the microbiome that underlie

336 community-level assembly processes. For instance, the recent iCAMP ${ }^{63}$ forms phylogenetic bins

337 of taxa, examines their phylogenetic and taxonomic turnover, and assigns the underlying

338 processes governing their turnover. Our analytical framework for identifying phylogenetic clades

339 under selection is conceptually similar to iCAMP and can be used in parallel with it. Like

340 iCAMP, our framework detects clades with distinctly different phylogenetic turnover than that

341 expected by chance. The detected phylogenetic clades do not necessarily need to have low

342 phylogenetic turnover like in the present study; clades with high phylogenetic turnover indicative

343 of heterogeneous selection (i.e., disproportionally present in different sample groups) can be

344 detected as well. Such patterns would indicate clades under selection in specific spatial or

345 temporal subsets depending on the study, and the assembly of the respective community pairs

346 should be governed by heterogeneous selection. Unlike iCAMP, however, our method avoids

347 phylogenetic binning and uses nearest-taxon phylogenetic distances. Both of these

348 methodological attributes can be valuable when examining patterns near the tips of the

349 phylogeny, such as microdiversity, which might not emerge with the use of other metrics ${ }^{20}$.

350 Therefore, our framework, rooted in community ${ }^{14}$ and metacommunity ${ }^{64}$ ecology, offers a novel

351 data-driven avenue that allows the exploration and quantification of the microdiversity

352 architecture of microbiomes without having to rely on isolates as often required 
353 previously ${ }^{9,11,12,65}$. Nevertheless, the short amplicon lengths used in most studies might not be

354 adequate to properly resolve the topology at the tips of the phylogeny. While this should have no

355 effect on the identification of phylogenetic clades with distinct z-scores compared to outgroups,

356 it might affect the identification of specific SVs and thus full-length 16S rRNA gene

357 amplicons ${ }^{66-68}$ might be used to construct phylogenetic trees with highly supported topologies

358 near the tips.

359 Contrary to the early expectations of an ecologically neutral origin of microdiversity

360 arising from genetic $\mathrm{drift}^{3}$, the link between selection and microdiversity that we show here

361 suggests that optimization of niche occupancy probably underlies the observed

362 microdiversification in GFS. This notion is supported by the presence of the same phylogenetic

363 groups that have different and microdiverse SVs in different GFS, probably corresponding to the

364 presence of different microniches therein. We conjecture that this could be a phenomenon

365 common to the microbiome of other extreme environments with reduced competitive exclusion

366 that might have been hitherto unrecognized because of the lack of adequate analytical

367 frameworks. The relaxation of the environmental extremeness owing to climate change may

368 change the balance among the selective processes in GFS and that could erode the microdiversity

369 of the GFS microbiome with yet unknown consequences for the overall biodiversity and

370 ecosystem functioning therein. 


\section{Methods}

\section{Sampling}

We sampled 20 glacier-fed streams at the Southern Alps in New Zealand along a 340-km

375 North-East - South-West transect (Fig. S1). The selected glaciers clustered in five major head

376 valley systems (Arthur's Pass, Westland, Mount Cook, Mount Aspiring and Milford Sound.

377 Glacier surface areas ranged between 0.5 to $35 \mathrm{~km}^{2}$, so that a wide range of runoff conditions

378 was encountered during sampling. We sampled stream bottom sediments from two reaches

379 within each of the 20 glacier-fed streams. The upper reaches (hereafter referred to as UP) were

380 located the closest possible to the glaciers' snouts. The lower reaches (hereafter referred to as

381 DN) were located 100 to $2500 \mathrm{~m}$ downstream, representing a gradient of decreasing connectivity

382 with the UP reaches in the same stream via the water flow. For operational purposes, we

383 assigned numbers to the sampled streams from 1 to 21, skipping number 4 (Fig. S1).

384 Within each reach, we sampled sediments from three different patches in order to assess

385 the within-reach variability. The patches were distanced 2-5 m apart. We sieved the wet sampled

386 sediments through two overlapping 315 and $250 \mu \mathrm{m}$ fire-sterilized sieves (Retsch, Woven Wire

387 Mesh Sieve - ø $200 \mathrm{~mm} / 203 \mathrm{~mm}$ ). We placed 30 grams of sediment in 10-ml cryovials (VWR)

388 and we flash froze them in liquid nitrogen for DNA extraction. For bacterial abundance analysis,

389 we filled 5-ml cryovials (VWR) with 2.5-3 grams of sediment containing a $10 \%$ solution of

390 paraformaldehyde/glutaraldehyde ${ }^{69}$ in $0.22 \mu \mathrm{m}$-filtered streamwater that we added in-situ, and

391 we flash froze the vials in liquid nitrogen. 
We measured stream temperature, dissolved oxygen and $\mathrm{pH}$ using a WTW Multi-

395 parameter portable meter (MultiLine ${ }^{\circledR}$ Multi 3630 IDS), conductivity using a WTW - IDS probe

396 (TetraCon ${ }^{\circledR}$ 925) and turbidity using a WTW portable turbidity meter (Turb® 430 IR). We

397 measured the concentration of chlorophyll $a$ in the sediment following a modified ethanol

398 extraction protocol as described elsewhere ${ }^{70}$. Geographical and physicochemical parameters are

399 shown in Table S5.

\section{Bacterial abundance}

402 We quantified the number of cells per gram of dry sediment using flow cytometry after

403 detaching the cells from the sediment matrix, by slightly modifying the method of Amalfitano \&

404 Fazi $^{71}$ as described elsewhere ${ }^{70}$. Briefly, we fixed 2.5 - 3 grams of wet sediment per sample in

405 situ in $1.8 \mathrm{ml}$ of filter-sterilized paraformaldehyde/glutaraldehyde solution ${ }^{69}$ within cryovials and

406 we flash-froze the vials in liquid nitrogen. At the end of the expedition, we transferred the

407 samples in dry ice back to the lab and we stored them at $-80^{\circ} \mathrm{C}$ until the analysis. To detach the

408 cells, we performed two rounds of mild shaking (Standard Analog Shaker, VWR, 15 min, 5.5

409 speed) followed by sonication (Sonifier 450, Branson, $1 \mathrm{~min}, 60 \%$ duty cycle, output 5) in $10 \mathrm{ml}$

410 of paraformaldehyde/glutaraldehyde solution supplemented with sodium pyrophosphate at a final

411 concentration of $0.025 \mathrm{mM}$. We pooled the resulting extracts per sample $(\sim 20-22 \mathrm{ml}$ in total),

412 mixed them thoroughly, transferred $1 \mathrm{ml}$ of each in a sterile $1.5 \mathrm{ml}$ tube and spinned them for 5

413 sec to pellet the large sediment particles. We then diluted $100 \mu$ of the supernatants 10 -fold in

414 paraformaldehyde/glutaraldehyde solution and we stained the dilutions with SybrGreen ${ }^{\circledR}$ (1X

415 final concentration, incubation for $15 \mathrm{~min}$ at $37^{\circ} \mathrm{C}$ ) before analyzing them on a NovoCyte flow

416 cytometer (ACEA Biosciences) equipped with a $488 \mathrm{~nm}$ laser. 
To analyze the stained samples we set the reading time to 2 min per sample and the flow

418 rate to $14 \mu \mathrm{l}$ per min, rinsing thrice and shaking once between samples. We identified and gated

419 the cell populations based on the height of their fluorescence signals on a 530/30 - 725/40 nm

420 biplot as previously described ${ }^{72}$ (Fig. S5), using the ACEA NovoExpress ${ }^{\circledR}$ software with

421 thresholds of 300 and 3000 on the front scatter and 530/30 nm channels, respectively. We

422 analyzed three stained technical replicates plus one unstained replicate of the same extract per

423 sample, the latter to exclude any background fluorescence. The coefficient of variation among

424 the counts from technical replicates was $7.5 \pm 5.1 \%$ on average. Finally, we corrected the acquired

425 numbers for the various dilution factors and for the sediments' water content (which we obtained

426 from the weight loss of oven-dried sediment samples) to obtain the amount of total cells per

427 gram of dry sediment.

DNA extraction, PCR amplification and 16S rRNA gene amplicon sequencing

430 We extracted DNA from sediment samples using a phenol-chloroform method with

431 certain modifications to address the nature of our samples ${ }^{73}$.We amplified the V3-V4

432 hypervariable regions of the bacterial $16 \mathrm{~S}$ rRNA gene using primers 341f (5'-

433 CCTACGGGNGGCWGCAG-3') and 785r (5'-GACTACHVGGGTATCTAATCC-3') ${ }^{74}$. Due to

434 low DNA yields and presence of inhibitors in the DNA extracts of certain samples and in an 435 attempt to avoid PCR biases due to unequal input DNA, we diluted all DNA samples to a final 436 concentration of $\leq 2-3 \mathrm{ng} / \mathrm{ul}$. The KAPA HiFi DNA Polymerase (Hot Start and Ready Mix 437 formulation) was used in a 25-ul-amplification reaction containing $1 \mathrm{X}$ PCR buffer, $1 \mathrm{uM}$ of 438 each primer, $0.48 \mathrm{ug} / \mathrm{ul} \mathrm{BSA}$ and $1.0 \mathrm{ul}$ of template DNA. Amplification was performed in a 439 Biometra Trio (Biometra) instrument. The thermal conditions applied after an initial denaturation 
440 at $95^{\circ} \mathrm{C}$ for $3 \mathrm{~min}$, were $94^{\circ} \mathrm{C}$ for $30 \mathrm{~s}, 55^{\circ} \mathrm{C}$ for $30 \mathrm{~s}$ and $72^{\circ} \mathrm{C}$ for $30 \mathrm{~s}$ for 25 cycles followed by

441 a final extension at $72^{\circ} \mathrm{C}$ for $5 \mathrm{~min}$. Amplification was verified on a $1.5 \%$ agarose gel and

442 products were sent to Lausanne Genomic Technologies Facility (Switzerland) for further

443 processing, library preparation and 300-base-pairs paired-end sequencing at an Illumina Miseq

444 platform.

\section{Sequence downstream analyses}

447 We used Trimmomatic v. $0.36^{75}$ for quality filtering of the sequencing reads. Shortly, we

448 truncated the reads in $4 \mathrm{bp}$ sliding windows at the first instance of mean quality dropping below a

449 Phred score of 15, we removed the three leading and trailing nucleotides and we discarded the 450 reads that were shorter than $200 \mathrm{bp}$.

451 We performed all subsequent sequence processing within the QIIME2 v.2019.1

452 framework $^{76}$. We used DADA2 ${ }^{77}$ with the default parameters to remove the primers, denoise and

453 join the reads into exact sequence variants (SVs). For this, 17 and 21 nucleotides (corresponding

454 to the primers' length) were removed at the beginning of the forward and reverse reads

455 respectively, and the reads were truncated at 300bp. We performed denoising and joining of the

456 reads using the default parameters, and we removed any SVs that were not found in at least two

457 samples. We used the alpha-rarefaction method implemented in the diversity plugin of QIIME2

458 to create the rarefaction curves (Fig. S6). We used the SV table that contained the raw sequence

459 counts of each SV at each sample to calculate the relative abundances of SVs within samples,

460 and we transformed the relative abundances into absolute abundances (cells per gram of dry

461 sediment) by multiplying with the cell counts derived from flow cytometry ${ }^{78}$. 
We assigned taxonomy with the feature-classifier plugin $^{79}$ in QIIME2. First, we trained

463 QIIME2's naïve Bayesian classifier using the fit-classifier-naïve-bayes method on the

464 Greengenes ${ }^{80} 99 \%$ OTUS database v. 13.5. We created this training set using the extract-reads

465 method with a minimal and maximal length of 250 and 550 nucleotides, respectively, and using

466 the primers' sequences. Finally, we assigned the taxonomy of the sequence variants using the

467 classify-sklearn method with default parameters. We considered the taxonomies down to the

468 genus level, ignoring "species" assignments that can be ambiguous based only on part of the 16S

469 rRNA gene ${ }^{81}$. At the Class level, Betaproteobacteria were dominant in all samples (Fig. S7). A

470 detailed taxonomic summary can be found in Supplementary Results.

471 To build the phylogenetic tree, we aligned the sequences of the SVs with mafft $t^{82}$ and we

472 trimmed the alignment with the mask method in QIIME2 using the default parameters. We then

473 used RAxML ${ }^{83}$ with the GTRCAT substitution model and the rapid bootstrap option to build the

474 tree, and the midpoint-root method to root the phylogenetic tree. To calculate pairwise

475 nucleotidic similarities we used ClustalOmega ${ }^{84}$ v.1.2.3.

\section{Identification of the core microbiome}

478 We identified the core microbiome across all samples based on taxonomy, i.e., as the 479 consensus taxonomic clades that are present in all 40 reaches (20 GFS x 2 reaches each). We 480 used the package metacoder ${ }^{85}$ in $\mathrm{R}^{86}$ to visualize the results as hierarchy trees.

\section{Multivariate statistics}

We used distance-based redundancy analysis to quantify the variance in the Bray-Curtis

484 similarity matrix (calculated using log-transformed absolute abundances) that could be explained 
485 by the measured physicochemical variables, using the capscale() function of the vegan ${ }^{87}$ package

486 in $\mathrm{R}$. We performed a stepwise forward selection based on the increase in the adjusted $\mathrm{R}^{2}$ to

487 select for the variables to include in the model, using the ordiR2step() function in vegan with 200

488 permutations (Table S6).

490 Quantification of the dominant assembly processes at the community level

491 We used the framework developed by Stegen and colleagues ${ }^{20,21}$ to quantify the dominant

492 assembly processes at the community level. This framework assigns differences between two

493 given communities to selection (either homogeneous or heterogeneous), to dispersal (either

494 homogenizing or limiting) or to the lack of any dominant process. The influence of selection is

495 first determined by examining the phylogenetic $\beta$-diversity via the z-score (in this case called $\beta$ -

496 nearest taxon index - $\beta$ NTI) of the observed $\beta$-mean nearest taxon distance $(\beta$-MNTD) from a

497 null distribution of the same metric. $\beta$ NTI scores less than -2 indicate that the observed $\beta$-MNTD

498 is significantly smaller than $\sim 95 \%$ of the null values and thus that homogeneous selection

499 between the compared communities causes them to have more similar species phylogenetically

500 (at short distances) than expected by chance. In analogy, $\beta$ NTI scores greater than +2 indicate the

501 dominance of heterogeneous selection. Community pairs with $\beta$ NTI scores between -2 and +2

502 are further compared in terms of composition using the Raup-Crick distances based on the Bray-

503 Curtis similarity $\left(\mathrm{RC}_{\mathrm{Bray}}\right)$, with the null distribution in this case being formed by probabilistic

504 permutations under weak selection and random dispersal. Here, values of $\mathrm{RC}_{\mathrm{Bray}}$ less than -0.95

505 and greater than 0.95 indicate less and more compositional turnover, respectively, than the null

506 expectation and that is attributed to homogenizing dispersal in the former case and to dispersal

507 limitation in the latter. 
509 a) some degree of migration occurred among local communities at least at some point in

510 evolutionary time and b) phylogenetic conservatism exists, i.e., phylogenetically more similar

511 organisms occupy more similar ecological niches. For our dataset, the first assumption probably

512 holds true even for the most distant GFS because at some point in geological time all the

513 sampled locations were covered under the same ice sheet. To test the second assumption of

514 phylogenetic conservatism we first calculated the niche optima of the SVs for each

515 physicochemical parameter that we included in the multivariate analyses (Table S6), as

516 previously described ${ }^{88}$, and we then calculated the niche distances among SVs as the euclidean

517 distance of their niche optima (after standardization of each parameter). We then performed a

518 mantel correlogram analysis, correlating the phylogenetic distances to the niche distances at

519 different distance classes. Proper use of the $\beta$ MNTD requires a positive correlation between the

520 two at short genetic distance classes, indicating that at short phylogenetic distances more related

521 SVs have shorter niche optima distances and therefore occupy more similar ecological niches;

522 that was indeed the case for our dataset (Figure S2). We calculated the abundance-weighted

$523 \beta$ MNTD using the comdistnt function of the picante ${ }^{89}$ package in $\mathrm{R}$ (setting abundance.weighted

$524=$ TRUE).

525

526 Identification of phylogenetic clades under homogeneous selection

527 In addition to inferring community-wise patterns of assembly using the framework of

528 Stegen et al. as described above, we further developed a framework to identify phylogenetic

529 clades under homogeneous selection. In analogy to the community-wise framework, we defined 
530 those clades as phylogenetically coherent units containing SVs with phylogenetically closer

531 relatives across communities than expected by chance.

532 Our method consists of the following steps:

533 1) For a given pair of communities and for each SV therein that is not present in both

534 communities, we calculate its z-score from a null distribution of log-transformed minimum

535 phylogenetic distances to assess how different its minimum phylogenetic distance is to that

536 expected by chance. For example, if we examine SV $x$ in the community pair $a-b$ and $x$ is present

537 in community $a$ and not in community $b$, we first find the minimum phylogenetic distance dist

538 between $x$ and all the SVs that are present in community $b$ and we log-transform it. We then

539 calculate a null distribution of minimum log-transformed phylogenetic distances between $x$ and

540 these SVs by randomly picking phylogenetic distances from the whole metacommunity distance

541 pool and assigning them to the SVs in the two communities, with null_mean and null_sd being

542 the mean and standard deviation of this distribution. Here, we performed 100 permutations to

543 calculate null_mean and null_sd, because this number was adequate to create normally

544 distributed null distances. For future studies we recommend that null distributions are checked

545 for normality depending on the sample size of each study and that the number of permutations is

546 adjusted accordingly. Finally, we calculate the z-score as:

547

548

$$
z \text {-score }=(\log (\text { dist })-\text { null_mean }) / \text { null_sd }
$$

550 2) We then calculate for each SV its total z-score across all community pairs. We use 551 phylofactorization ${ }^{29,30}$ to identify phylogenetically coherent groups of SVs with significantly 
552 different total scores compared to outgroups and to extract the consensus taxonomic 553 classification of the SVs within.

554 3) We also detect SVs with constantly low phylogenetic turnover (LPT-SVs). For that, 555 we calculate the median z-score and we classify LPT-SVs using a threshold of significance based

556 on a median z-score less than -2 . We perceive this threshold as an indication that a given SV is

557 consistently under homogeneous selection, because this SV has phylogenetically nearest

558 neighboring SVs with shorter distances than those expected by chance in at least $50 \%$ of the

559 applicable community pairs (i.e., those community pairs where the SV is found in exactly one of

560 the two communities). While this classification was tailored for the purposes of our study, the

561 concept can be expanded in future studies to potentially detect and classify SVs based on other

562 numerical threshold or even SVs with higher phylogenetic turnover than that expected by

563 chance. In the latter case the numerical threshold should be positive and it depends on the study

564 to find appropriate criteria. If, for instance, heterogeneous selection is detected at the community

565 level for a subset of community pairs, a threshold of +2 regarding the median could be used to

566 detect SVs with consistently high phylogenetic turnover among the majority of these community

567 pairs. 


\section{References}

5691 Larkin, A. A. \& Martiny, A. C. Microdiversity shapes the traits, niche space, and 570 biogeography of microbial taxa. Environ. Microbiol. Rep. 9, 55-70 (2017).

5712 Acinas, S. G. et al. Fine-scale phylogenetic architecture of a complex bacterial community. Nature 430, 551-554 (2004). Thompson, J. R. et al. Genotypic diversity within a natural coastal bacterioplankton population. Science 307, 1311 (2005).

5754 Thrash, C. J. et al. Single-cell enabled comparative genomics of a deep ocean SAR11

576 bathytype. ISME J. 8, 1440-1451 (2014). Hunt, D. E. et al. Resource partitioning and sympatric differentiation among closely related bacterioplankton. Science 320, 1081 (2008).

5796 Kent, A. G. et al. Parallel phylogeography of Prochlorococcus and Synechococcus. ISME $580 \quad$ J. 13, 430-441 (2019).

5817 Brown, M. V. \& Furham, J. A. Marine bacterial microdiversity as revealed by internal

582 transcribed spacer analysis. Aquat. Microb. Ecol. 41, 15-23 (2005). Scanlan, D. J. et al. Ecological genomics of marine picocyanobacteria. Microbiol. Mol. Biol. Rev. 73, 249 (2009).

585 Yung, C.-M. et al. Thermally adaptive tradeoffs in closely related marine bacterial strains. Environ. Microbiol. 17, 2421-2429 (2015). specific microdiversification in the ubiquitous and abundant freshwater genus Limnohabitans. Appl. Environ. Microbiol. 86, e00140-00120 (2020).

591

592

593

594

595 Chase, A. B. et al. Microdiversity of an abundant terrestrial bacterium encompasses extensive variation in ecologically relevant traits. mBio 8 (2017).

12 Choudoir, M. J. \& Buckley, D. H. Phylogenetic conservatism of thermal traits explains dispersal limitation and genomic differentiation of Streptomyces sister-taxa. ISME J. 12, 2176-2186 (2018).

596 Martiny, J. B., Jones, S. E., Lennon, J. T. \& Martiny, A. C. Microbiomes in light of traits: A phylogenetic perspective. Science 350, 9323 (2015). Vellend, B. M. Conceptual synthesis in community ecology. Quart. Rev. Biol. 85, 183206 (2010). Chafee, M. et al. Recurrent patterns of microdiversity in a temperate coastal marine environment. ISME J. 12, 237-252 (2018). among marine phytoplankton, bacteria and myoviruses shows microdiversity matters. ISME J. 11, 1614-1629 (2017). Garcia-Garcia, N., Tamames, J., Linz, A. M., Pedros-Alio, C. \& Puente-Sanchez, F. Microdiversity ensures the maintenance of functional microbial communities under changing environmental conditions. ISME J. 13, 2969-2983 (2019). Tromas, N. et al. The evolution of realized niches within freshwater Synechococcus. Environ. Microbiol. 22, 1238-1250 (2020).

610 Konstantinidis, K. T., Ramette, A. \& Tiedje, J. M. The bacterial species definition in the genomic era. Philos. Trans. R. Soc. B: Biol. Sci. 361, 1929-1940 (2006). that impose them. ISME J. 7, 2069-2079 (2013). 
61321 Stegen, J. C., Lin, X., Fredrickson, J. K. \& Konopka, A. E. Estimating and mapping ecological processes influencing microbial community assembly. Front. Microbiol. 6 (2015).

22 Allen, R., Hoffmann, L. J., Larcombe, M. J., Louisson, Z. \& Summerfield, T. C. Homogeneous environmental selection dominates microbial community assembly in the oligotrophic South Pacific Gyre. Mol. Ecol. 29, 4680-4691, (2020).

$23 \mathrm{Li}$, Y. et al. Homogeneous selection dominates the microbial community assembly in the sediment of the Three Gorges Reservoir. Sci. Tot. Environ. 690, 50-60 (2019).

24 Zhang, K. et al. Salinity is a key determinant for soil microbial communities in a desert ecosystem. mSystems 4 (2019).

25 Cauvy-Fraunié, S. \& Dangles, O. A global synthesis of biodiversity responses to glacier retreat. Nat. Ecol. Evol. 3, 1675-1685 (2019).

26 Wilhelm, L., Singer, G. A., Fasching, C., Battin, T. J. \& Besemer, K. Microbial biodiversity in glacier-fed streams. ISME J. 7, 1651 (2013).

27 Milner, A. M. et al. Glacier shrinkage driving global changes in downstream systems. Proc. Nat. Acad. Sci. USA 114, 9770 (2017).

28 Fodelianakis, S. et al. Dispersal homogenizes communities via immigration even at low rates in a simplified synthetic bacterial metacommunity. Nat. Commun. 10, 1314, (2019). Washburne, A. D. et al. Phylogenetic factorization of compositional data yields lineagelevel associations in microbiome datasets. PeerJ 5, e2969 (2017).

30 Washburne, A. D. et al. Phylofactorization: a graph partitioning algorithm to identify phylogenetic scales of ecological data. Ecol. Monogr. 89, e01353, (2019).

31 Logares, R. et al. Biogeography of bacterial communities exposed to progressive longterm environmental change. ISME J. 7, 937-948 (2013).

32 Cerqueira, T., Barroso, C., Froufe, H., Egas, C. \& Bettencourt, R. Metagenomic signatures of microbial communities in deep-sea hydrothermal sediments of Azores Vent Fields. Microb. Ecol. 76, 387-403 (2018). continental deep subsurface: Sanford underground research facility (SURF), USA. Front. Microbiol. 5 (2014).

34 Tran, P. et al. Microbial life under ice: Metagenome diversity and in situ activity of Verrucomicrobia in seasonally ice-covered Lakes. Environ. Microbiol. 20, 2568-2584 (2018).

35 Vick-Majors, T. J., Priscu, J. C. \& Amaral-Zettler, L. A. Modular community structure suggests metabolic plasticity during the transition to polar night in ice-covered Antarctic lakes. ISME J. 8, 778-789 (2014). distribution of Polaromonas phylotypes - evidence for a highly successful dispersal capacity. PloS One 6, e23742 (2011).

653

37 Smith, H. J., Foreman, C. M. \& Ramaraj, T. Draft genome sequence of a metabolically diverse Antarctic supraglacial stream organism, Polaromonas sp. strain CG9_12, determined using Pacific Biosciences single-molecule real-time sequencing technology. Genome Announc. 2, e01242-01214 (2014). 
Rime, T., Hartmann, M. \& Frey, B. Potential sources of microbial colonizers in an initial soil ecosystem after retreat of an alpine glacier. ISME J. 10, 1625-1641 (2016).

40 Liu, Q., Zhou, Y.-G. \& Xin, Y.-H. High diversity and distinctive community structure of bacteria on glaciers in China revealed by 454 pyrosequencing. Syst. Appl. Microbiol. 38, 578-585 (2015).

41 Kalyuzhnaya, M. G., Bowerman, S., Lara, J. C., Lidstrom, M. E. \& Chistoserdova, L. Methylotenera mobilis gen. nov., sp. nov., an obligately methylamine-utilizing bacterium within the family Methylophilaceae. Int. J. Syst. Evol. Microbiol. 56, 2819-2823 (2006). Kane, S. R. et al. Whole-genome analysis of the methyl tert-butyl ether-degrading BetaProteobacterium Methylibium petroleiphilum PM1. J. Bacteriol. 189, 1931, (2007). Martineau, C., Mauffrey, F., Villemur, R. \& Müller, V. Comparative analysis of denitrifying activities of Hyphomicrobium nitrativorans, Hyphomicrobium denitrificans, and Hyphomicrobium zavarzinii. Appl. Environ. Microbiol. 81, 5003-5014 (2015). Dieser, M. et al. Molecular and biogeochemical evidence for methane cycl
the western margin of the Greenland Ice Sheet. ISME J. 8, 2305-2316 (2014). Michaud, A. B. et al. Microbial oxidation as
Ice Sheet. Nat. Geosci. 10, 582-586 (2017). Baker, J. M. et al. Genome sequence of Rhodoferax antarcticus ANT.BRT; a psychrophilic purple nonsulfur bacterium from an Antarctic microbial mat. Microorganisms 5, (2017). degradation potential of a cold-adapted oil/PAH-degrading marine bacterial consortium from Kongsfjorden (Arctic region). Rendiconti Lincei 27, 261-270 (2016).

Zhong, Z.-P. et al. Clean low-biomass procedures and their application to ancient ice core microorganisms. Front. Microbiol. 9 (2018).

Bai, Y. et al. Variation in denitrifying bacterial communities along a primary succession in the Hailuogou Glacier retreat area, China. PeerJ 7:e7356 (2019).

Garcia-Lopez, E., Rodriguez-Lorente, I., Alcazar, P. \& Cid, C. Microbial communities in coastal glaciers and tidewater tongues of Svalbard archipelago, Norway. Front. Mar. Sci. 5 (2019).

52 Harrold, Z. R. et al. Aerobic and anaerobic thiosulfate oxidation by a cold-adapted, subglacial chemoautotroph. Appl. Environ. Microbiol. 82, 1486-1495 (2016).

53 Franzetti, A. et al. Early ecological succession patterns of bacterial, fungal and plant communities along a chronosequence in a recently deglaciated area of the Italian Alps. FEMS Microbiol. Ecol. 96 (2020).

4 Kohler, T. J., Van Horn, D. J., Darling, J. P., Takacs-Vesbach, C. D. \& McKnight, D. M. Nutrient treatments alter microbial mat colonization in two glacial meltwater streams from the McMurdo Dry Valleys, Antarctica. FEMS Microbiol. Ecol. 92:4 (2016).

55 Sawayama, M. et al. Isolation of a Leptothrix strain, OUMS1, from ocherous deposits in groundwater. Cur. Microbiol. 63, 173-180 (2011).

$\mathrm{Li}$, Y. et al. Reconstruction of the functional ecosystem in the high light, low temperature union glacier region, Antarctica. Front. Microbiol. 10 (2019).

57 Milner, A. M. et al. Glacier shrinkage driving global changes in downstream systems. Proc. Nat. Acad. Sci. USA 114, 9770-9778 (2017). 
58 Jorquera, M. A., Graether, S. P. \& Maruyama, F. Editorial: bioprospecting and biotechnology of extremophiles. Front. Bioeng. Biotech. 7, 204 (2019).

59 Stegen, J. C., Lin, X., Konopka, A. E. \& Fredrickson, J. K. Stochastic and deterministic assembly processes in subsurface microbial communities. ISME J. 6, 1653-1664 (2012).

60 Zhou, J. \& Ning, D. Stochastic community assembly: does it matter in microbial ecology? Microbiol. Mol. Biol. Rev. 81(4) (2017).

61 Ning, D., Deng, Y., Tiedje, J. M. \& Zhou, J. A general framework for quantitatively assessing ecological stochasticity. Proc. Nat. Acad. Sci. USA 116, 16892-16898 (2019).

62 Zhou, J. et al. Stochasticity, succession, and environmental perturbations in a fluidic ecosystem. Proc. Nat. Acad. Sci. USA 111, E836-845 (2014).

63 Ning, D. et al. A quantitative framework reveals ecological drivers of grassland microbial community assembly in response to warming. Nat. Commun. 11, 4717 (2020).

64 Leibold, M. A. et al. The metacommunity concept: a framework for multi-scale community ecology. Ecol. Let. 7, 601-613 (2004).

65 Chase, A. B. et al. Emergence of soil bacterial ecotypes along a climate gradient. Environ. Microbiol. 20, 4112-4126 (2018).

66 Callahan, B. J., Grinevich, D., Thakur, S., Balamotis, M. A. \& Yehezkel, T. B. Ultraaccurate microbial amplicon sequencing rirectly from complex samples with synthetic long reads. bioRxiv, (2020).

67 Matsuo, Y. et al. Full-length 16S rRNA gene amplicon analysis of human gut microbiota using MinION ${ }^{\mathrm{TM}}$ nanopore sequencing confers species-level resolution. bioRxiv (2020).

68 Nygaard, A. B., Tunsjø, H. S., Meisal, R. \& Charnock, C. A preliminary study on the potential of Nanopore MinION and Illumina MiSeq 16S rRNA gene sequencing to characterize building-dust microbiomes. Sci. Rep. 10, 3209 (2020).

69 Duarte, C. M. et al. Discovery of Afifi, the shallowest and southernmost brine pool reported in the Red Sea. Sci. Rep. 10, 910 (2020).

70 Kohler, T. J. et al. Patterns and drivers of extracellular enzyme activity in New Zealand glacier-fed streams. Front. Microbiol. 11, 2922 (2020).

71 Amalfitano, S. \& Fazi, S. Recovery and quantification of bacterial cells associated with streambed sediments. J. Microbiol. Methods 75, 237-243 (2008).

72 Hammes, F. et al. Flow-cytometric total bacterial cell counts as a descriptive microbiological parameter for drinking water treatment processes. Water Res. 42, 269277 (2008).

73 Busi, S. B. et al. Optimised biomolecular extraction for metagenomic analysis of microbial biofilms from high-mountain streams. PeerJ 8, e9973, (2020).

74 Klindworth, A. et al. Evaluation of general 16S ribosomal RNA gene PCR primers for classical and next-generation sequencing-based diversity studies. Nucleic Acids Res. 41(1), e1 (2013).

75 Bolger, A. M., Lohse, M. \& Usadel, B. Trimmomatic: a flexible trimmer for Illumina sequence data. Bioinformatics 30, 2114-2120 (2014).

76 Bolyen, E. et al. Reproducible, interactive, scalable and extensible microbiome data science using QIIME 2. Nat. Biotech. 37, 852-857 (2019).

77 Callahan, B. J. et al. DADA2: High-resolution sample inference from Illumina amplicon data. Nat. Meth. 13, 581-583 (2016).

78 Props, R. et al. Absolute quantification of microbial taxon abundances. ISME J. 11, 584587 (2017). 
75179 Bokulich, N. A. et al. Optimizing taxonomic classification of marker-gene amplicon sequences with QIIME 2's q2-feature-classifier plugin. Microbiome 6, 90 (2018).

753 DeSantis, T. Z. et al. Greengenes, a chimera-checked 16S rRNA gene database and workbench compatible with ARB. Appl. Environ. Microbiol. 72, 5069-5072 (2006). Singer, E. et al. High-resolution phylogenetic microbial community profiling. ISME J. 10, 2020-2032 (2016). Katoh, K. \& Standley, D. M. MAFFT multiple sequence alignment software version 7: improvements in performance and usability. Mol. Biol. Evol. 30, 772-780 (2013). Stamatakis, A. RAxML version 8: a tool for phylogenetic analysis and post-analysis of large phylogenies. Bioinformatics 30, 1312-1313 (2014).

84 Sievers, F. et al. Fast, scalable generation of high-quality protein multiple sequence alignments using Clustal Omega. Mol. Syst. Biol. 7, 539-539 (2011).

85 Foster, Z. S. L., Sharpton, T. J. \& Grünwald, N. J. Metacoder: An R package for

86 R: A Language and Environment for Statistical Computing (R Foundation for Statistical visualization and manipulation of community taxonomic diversity data. PLOS Comput. Computing, Vienna, Austria, 2014).

89 Kembel, S. W. et al. Picante: R tools for integrating phylogenies and ecology. Bioinformatics 26, 1463-1464 (2010). 


\section{Acknowledgements}

779 granted to TJB. The funders had no role in study design, data collection and analysis, decision to

780 publish, or preparation of the manuscript.

\section{Author contributions}

784 sampling; PP, HP, TK, JB, MSch, SB and SF performed lab work; SF and MB analyzed the

785 sequencing data; AW and SF developed new code; TJB and PW provided resources; SF, TJB

786 and HP wrote the manuscript with input from all authors.

\section{Data Availability}

790 number PRJEB40567.

\section{Code Availability}

794 (https://github.com/sfodel/phylo_z_scores).

796 Conflict of Interest Statement 